\title{
NR4A3, a possibile oncogenic factor for neuroblastoma associated with CpGi methylation within the third exon
}

\author{
SHOTA UEKUSA $^{1,2^{*}}$, HIROYUKI KAWASHIMA ${ }^{1,2^{*}}$, KIMINOBU SUGITO $^{1}$, SHINSUKE YOSHIZAWA ${ }^{1,2}$, \\ YUI SHINOJIMA ${ }^{2}$, JUN IGARASHI ${ }^{2,3}$, SRIMOYEE GHOSH ${ }^{4}$, XAOFEI WANG ${ }^{2,3,5}$, KYOKO FUJIWARA ${ }^{2,6,7}$, \\ TARO IKEDA $^{1}$, TSUGUMICHI KOSHINAGA ${ }^{1}$, MASAYOSHI SOMA ${ }^{6,7}$ and HIROKI NAGASE ${ }^{2,3,5,7}$
}

\author{
Departments of ${ }^{1}$ Pediatric Surgery and ${ }^{2}$ Cancer Genetics, School of Medicine, ${ }^{3}$ Life Science Advanced Research Institute \\ for the Sciences and Humanities, Nihon University, Tokyo, Japan; ${ }^{4}$ Department of Zoology, North-Eastern Hill University, \\ Meghalaya, India; ${ }^{5}$ Chiba Cancer Center Research Institute, Chiba; ${ }^{6}$ Innovative Therapy Research Group, \\ Nihon University Research Institute of Medical Science, ${ }^{7}$ Division of General Medicine, \\ Department of Medicine, Nihon University School of Medicine, Tokyo, Japan
}

Received December 18, 2013; Accepted February 12, 2014

DOI: 10.3892/ijo.2014.2340

\begin{abstract}
Aberrant methylation of $\mathrm{Nr} 4 a 3$ exon $3 \mathrm{CpG}$ island (CpGi) was initially identified during multistep mouse skin carcinogenesis. $\mathrm{Nr} 4 \mathrm{a} 3$ is also known as a critical gene for neuronal development. Thus, we examined the $\mathrm{Nr} 4 \mathrm{a} 3 \mathrm{exon} 3$ CpGi methylation in mouse brain tissues from 15-day embryos, newborns and 12-week-old adults and found significant increase of its methylation and $\mathrm{Nr} 4 \mathrm{a} 3$ expression during mouse brain development after birth. In addition, homologous region in human genome was frequently and aberrantly methylated in neuroblastoma specimens. A quantitative analysis of DNA methylation revealed that hypomethylation of $\mathrm{CpG}$ islands on NR4A3 exon 3, but not on exon 1 was identified in three neuroblastomas compared with matched adrenal glands. Additional analysis for 20 neuroblastoma patients was performed and 8 of 20 showed hypomethylation of the
\end{abstract}

Correspondence to: Professor Hiroki Nagase, Chiba Cancer Center Research Institute, 666-2 Nitona-Cho, Chuo-Ku, Chiba 260-8717, Japan

E-mail: hnagase@chiba-cc.jp

${ }^{*}$ Contributed equally

Abbreviations: T-DMRs, tissue-specific differentially methylated regions; DS-DMRs, developmental stage differentially methylated regions; $\mathrm{CpGi}$, CpG island; E15, 15-day-old embryo; NB, new born; AD, 12-week adult; TrkA, tropomyosin receptor kinase A; NGF, nerve growth factor; hMC, homogeneous MassCLEAVE; MALDI-TOF MS, matrix-assisted laser desorption/ionization time-of-flight mass spectrometry; Tm, melting temperature; PCR, polymerase chain reaction; TBS, Tris-buffered saline; PBS, phosphate-buffered saline; CTCF, CCCTC binding factor; UTR, untranslated region; AUF1, AU-rich element binding factor 1; HUR, Hu antigen R

Key words: nuclear receptor subfamily 4, group A, member 3, DNA methylation, neuroblastoma
CpGi on NR4A3 exon 3. The survival rate of those 8 patients was significantly lower compared with those in patients with hypermethylation. Immunohistochemical NR4A3 expression was generally faint in neuroblastoma tissues compared with normal tissues. Moreover, the MYCN amplified NB9 cell line showed hypomethylation and low expression of NR4A3, while the non-MYCN amplified NB69 cell line showed hypermethylation and high expression. These results indicate that DNA hypomethylation of the $\mathrm{CpGi}$ at $N R 4 A 3$ exon 3 is associated with low NR4A3 expression, and correlates with poor prognosis of neuroblastoma. Since NR4A3 upregulation associated with the hypermethylation and neuronal differentiation in mice, poor prognosis of neuroblastoma associated with NR4A3 low expression may be partly explained by dysregulation of its differentiation.

\section{Introduction}

Neuroblastoma is an embryonic tumor of neuroectodermal cells derived from the neural crest. It is the most common extracranial solid tumor in children, and it accounts for approximately $15 \%$ of all pediatric oncology death. Survival rate of the patient with high-risk neuroblastoma is still $<40 \%$, despite combined modality therapy $(1,2)$.

Recent advances have disclosed the significance of epigenetic events in the development and progression of human tumorigenesis. Generally, global DNA methylation levels are low in cancer and has been linked to genomic instability, which can lead to DNA damage. On the other hand, promoter-specific hypermethylation of specific genes, such as tumor suppressor genes, is the most common event in tumorigenesis (3). It is also reported that predetermined epigenetic program provides required direction for the number of changes during embryonic and postnatal development that are necessary for proceeding from an oocyte to a fully developed adult animal (4). DNA methylation is the one of the best-characterized epigenetic modifications and plays an important role in the diverse genomic processes, 
Table I. The tissue samples analyzed.

\begin{tabular}{|c|c|c|c|c|c|c|c|}
\hline Patient & $\begin{array}{l}\text { Age at diagnosis } \\
\text { (month) }\end{array}$ & INSS & INPC & $\begin{array}{l}\text { Shimada } \\
\text { classification }\end{array}$ & $\begin{array}{l}\text { Copy nos. } \\
\text { of } M Y C N\end{array}$ & $\begin{array}{l}\text { Prognosis } \\
\text { (month) }\end{array}$ & $\begin{array}{l}\text { Methylation level of } \\
N R 4 A 3 \text { exon } 3(\%)^{\mathrm{a}}\end{array}$ \\
\hline Case 1 & 6 & 1 & NBL, D & FH & 1 & $48 \mathrm{~S}$ & $43.1 \pm 5.9$ \\
\hline Case 2 & 10 & 2 & NBL, PD & FH & 1 & $26 \mathrm{~S}$ & $67.1 \pm 2.5$ \\
\hline Case 3 & 6 & 2 & NBL, PD & FH & 1 & $48 \mathrm{~S}$ & $20.1 \pm 0.9$ \\
\hline Case 4 & 6 & $4 \mathrm{~S}$ & NBL, UD & FH & 1 & $48 \mathrm{~S}$ & $43.1 \pm 5.9$ \\
\hline Case 5 & 6 & 2 & NBL, PD & $\mathrm{FH}$ & 1 & $48 \mathrm{~S}$ & $7.6 \pm 0.1$ \\
\hline Case 6 & 7 & 2 & NBL, PD & FH & 1 & $48 \mathrm{~S}$ & $18.4 \pm 1.6$ \\
\hline Case 7 & 8 & 2 & NBL, PD & FH & 1 & $48 \mathrm{~S}$ & $83.2 \pm 0.4$ \\
\hline Case 8 & 26 & 4 & NBL, PD & UH & 10 & $6 \mathrm{R}$ & $36.1 \pm 1.5$ \\
\hline Case 9 & 36 & 4 & NBL, UD & $\mathrm{UH}$ & 20 & $48 \mathrm{~S}$ & $-0.4 \pm 8.9$ \\
\hline Case 10 & 30 & 4 & NBL, PD & $\mathrm{UH}$ & 1 & $48 \mathrm{~S}$ & $34.3 \pm 2.4$ \\
\hline Case 11 & 47 & 4 & NBL, UD & $\mathrm{UH}$ & 20 & $4 \mathrm{R}$ & $8.5 \pm 1.5$ \\
\hline Case 12 & 21 & 3 & NBL, UD & UH & 150 & $7 \mathrm{R}$ & $2.1 \pm 7.3$ \\
\hline Case 13 & 98 & 3 & NBL, UD & UH & 1 & $48 \mathrm{~S}$ & $-1.2 \pm 7.3$ \\
\hline Case 14 & 77 & 4 & NBL, PD & UH & 3 & $4 \mathrm{~S}$ & $14.7 \pm 5.9$ \\
\hline Case 15 & 79 & 4 & NBL, PD & UH & 3 & $35 R$ & $39.1 \pm 2.2$ \\
\hline Case 16 & 53 & 4 & NBL, UD & UH & 4 & $24 R$ & $79.4 \pm 0.6$ \\
\hline Case 17 & 20 & 4 & NBL, UD & $\mathrm{UH}$ & 81 & $5 \mathrm{R}$ & $9.1 \pm 3.8$ \\
\hline Case 18 & 68 & 4 & NBL, PD & UH & 1 & $10 \mathrm{R}$ & $5.2 \pm 2.5$ \\
\hline Case 19 & 18 & 4 & NBL, PD & UH & 119 & $37 \mathrm{~S}$ & $5.4 \pm 4.1$ \\
\hline Case 20 & 33 & 4 & NBL, UD & UH & 1 & $36 \mathrm{~S}$ & $25.6 \pm 10.1$ \\
\hline
\end{tabular}

INSS, International Neuroblastoma Staging System; INPC, International Neuroblastoma Pathology Committee; NBL, neuroblastoma; PD, poorly differentiated; UD, undifferentiated; FH, favorable histology; $\mathrm{UH}$, unfavorable histology; S, recurrent free survival; R, recurrent. ${ }^{\mathrm{a} D a t a}$ are shown as mean $\pm \mathrm{SD}$.

such as gene regulation, chromosomal stability, parental imprinting and X-inactivation (5). Recent genome-wide DNA methylation searches indicate that 4 to $17 \%$ of $\mathrm{CpG}$ sites are different in methylation among tissues and developmental processes (6-8). The methylation status at the tissue-specific differentially methylated regions (T-DMRs) and developmental-specific differentially methylated regions (DS-DMRs) are suggested to play important roles in development and differentiation.

Nuclear receptor subfamily 4, group A, member 3 ( $N r 4 a 3)$, also known as neuron-derived orphan receptor 1 (NOR1), is a member of NR4A subgroup of orphan nuclear receptor. In mammals, the NR4A subgroup consists of NR4A1 (Nur77), NR4A2 (Nurr1) and NR4A3 (9). The monomer form of those receptors binds to the nerve growth factor-induced clone $\mathrm{B}$ response element (NBRE), and homodimer or heterodimer forms bind to the Nur1 response element in the promoter of their target genes which may be essential for the development of dopaminergic neurons in the midbrain (10). We identified the CpG sites in Nr4a3 exon 3 as a mouse skin cancer T-DMR and a mouse brain DS-DMR by using analyses of restriction landmark genomic scanning (RLGS) and methyl-DNA immunoprecipitation (MeDIP), respectively (Fujiwara et al, unpublished data). Here, we analyzed involvement of DNA methylation at $\mathrm{Nr} 4 \mathrm{a} 3$ exon $3 \mathrm{CpGi}$ in $\mathrm{Nr} 4 \mathrm{a} 3$ expression, mouse brain development, neuroblastomagenesis and association with its poor prognosis.

\section{Materials and methods}

Tissue samples. C57 BL/6J mice were purchased from Jackson Laboratory (Bar Harbor, ME) and maintained in Oriental Yeast Co. Ltd (Tokyo, Japan). Brain specimens from mice at three different developmental stages: E15, 15-day-old embryo; NB, new born; and AD, 12-week adult; were disected and stored as described previously (11).

Twenty primary neuroblastoma tumors were obtained in Nihon University Hospital (Tokyo, Japan) at the time of diagnosis, from 1999 to 2007. All the analyses of those specimens were performed under the approval of Nihon University Institutional Review Boards (IRB no. 51). Neither neoadjuvant chemotherapy nor irradiation therapy was given preoperatively to any patient. Four adrenal samples were collected from a nephroblastoma patient undergoing nephrectomy and from 3 neuroblastoma patients (cases 3,8 and 20) undergoing tumor resection. All of the samples were immediately snap-frozen in liquid nitrogen and stored at $-80^{\circ} \mathrm{C}$ until use. Summary of these patients is shown in Table I.

Cell lines and culture condition. Human neuroblastoma cell lines NB9 and NB69 were obtained from Riken Cell Bank (Tsukuba, Japan). Both human neuroblastoma cell lines were maintained in RPMI-1640 (Nalarai Tesque, Kyoto, Japan) supplemented with $15 \%$ fetal bovine serum (Nichirei Biosciences, Tokyo, Japan), $100 \mathrm{IU} / \mathrm{ml}$ penicillin $\left(\mathrm{Gibco}^{\mathrm{TM}}\right.$, 
Table II. The primers for quantitative DNA methylation analysis.

\begin{tabular}{lll}
\hline Primer name & & \multicolumn{1}{c}{ Sequence } \\
\hline NR3A3-a & Forward & GGAAATTGTTAAGTGTTTTTTTATAT \\
& Reverse 1 & CAACCACCACTTCCTAAAT \\
& Reverse 2 & CGACCACCACTTCCTAAAT \\
NR3A3-b & Forward & AGTTTTAGAATTTATGTAAGAGGAAAG \\
& Reverse 1 & CACCCAACTATCAAACTC \\
NR3A3-c & Reverse 2 & CGCCCAACTATCAAACTC \\
& Forward & GAGGTGTTGTTTAGTATTTTTATGTATTTTAAGTAG \\
& Reverse & CTCACCTTAAAAAAACCCTTACAACC \\
\hline
\end{tabular}

Each reverse primer has a T7-promotor tag ( $5^{\prime}$-cagtaatacgactcactatagggagaaggct-3') for in vitro transcription and the forward primer is tagged with a 10 mer tag (5'-aggaagagag-3') to balance Tm.

Carlsbad, CA) and $100 \mu \mathrm{l} / \mathrm{ml}$ streptomycin (Gibco). The cells were cultured in a $37^{\circ} \mathrm{C}$ humidified atmosphere containing $5 \% \mathrm{CO}_{2}$ maintained in appropriate conditions recommended by the manufacturers.

DNA preparation and bisulfite treatment. Total genomic DNA was extracted from mouse brains, primary tumors, neuroblastoma cell lines and normal adrenal medullas with DNeasy Tissue Kit (Qiagen, Valencia, CA) and modified by sodium bisulfite using the EZ DNA Methylation Kit (Zymo Research, Orange, CA), by following the manufacturer's instructions.

Quantitative analysis of DNA methylation using base-specific cleavage and matrix-assisted laser desorption/ionization time-of-flight mass spectrometry (MALDI-TOF MS). Sequenom MassARRAY quantitative methylation analysis (12) using the MassARRAY Compact System (www. sequenom.com) was employed for the quantitative DNA methylation analysis at $\mathrm{CpG}$ dinucleotides. This system utilizes mass spectrometry (MS) for the detection and quantitative analysis of DNA methylation using homogeneous MassCLEAVE (hMC) base-specific cleavage and matrixassisted laser desorption/ionization time-of-flight (MALDI-TOF) MS (13). The MethPrimer program (http:// www.urogene.org/methprimer/index1.html) (12) was used to design bisulfite PCR primers (Table II). Each reverse primer has a T7-promotor tag for in vitro transcription (5'-cagtaatacgactcactatagggagaaggct-3'), and the forward primer is tagged with a 10 mer to balance melting temperature (TM) (5'-aggaagagag-3'). All primers were purchased from Operon (Tokyo, Japan). Polymerase chain reaction (PCR) amplification was performed using HotStarTaq Polymerase (Qiagen) in a $5 \mu \mathrm{l}$ reaction volume using PCR primers at a $200 \mathrm{nM}$ final concentration, and bisulfate treated DNA $(\sim 20 \mathrm{ng} / \mathrm{ml})$. After the treatment of shrimp alkaline phosphatase, $2 \mu \mathrm{l}$ of the PCR products was used as a template for in vitro transcription and RNase A Cleavage for the T-reverse reaction ( 3 ' to either rUTP or rCTP), as described in the manufacturer's instructions (Sequenom hMC, Sequenom, San Diego, CA). The samples were desalted and spotted on a 384-pad SpectroCHIP (Sequenom) using a MassARRAY nanodispenser (Samsung Seoul, Korea), followed by spectral acquisition on a MassARRAY Analyzer Compact MALDITOF MS (Sequenom). The resultant methylation calls were analyzed by EpiTYPER software v1.0 (Sequenom) to generate quantitative measurements for each $\mathrm{CpG}$ site or an aggregate of multiple $\mathrm{CpG}$ sites. Since maldi-TOF mass methylated peaks do not denote a particular $\mathrm{CpG}$ site, but rather corresponds to the number of $\mathrm{CpG}$ sites methylated within the cleavage fragment, we decided to present average percent methylation of all $\mathrm{CpG}$ sites in the bisulfite PCR fragment with the standard curve.

Standard curve of DNA methylation level was made by using $0,25,50,75$ and $100 \%$ methylated samples. BAC DNA (RPMI-11 341L6) obtained from Roswell Park Cancer Institute (Buffalo, NY) was used as $0 \%$ methylation and M.Sss-1 double treated BAC DNA was used as $100 \%$ methylation. The PCR was carried out with a final volume of $50 \mu \mathrm{l}$, containing $1.0 \mu \mathrm{l}$ of each $10.0 \mu \mathrm{M}$ primer (final concentration $0.2 \mu \mathrm{M}), 8.0 \mu \mathrm{l}$ of $2.5 \mathrm{mM}$ dNTP, $25 \mu \mathrm{l}$ of 2 times GC Buffer (Takara Bio, Shiga, Japan), 0.5 U of LA taq (Takara Bio) and $1 \mu 1$ of genomic DNA as a template. Amplification was carried out with an initial denaturing at $94^{\circ} \mathrm{C}$ for $1 \mathrm{~min}$ followed by 45 cycles of denaturing at $94^{\circ} \mathrm{C}$ for $30 \mathrm{sec}$, annealing for $1 \mathrm{~min}$ at the annealing temperature of each primer $\left(60^{\circ} \mathrm{C}\right)$, extension for $3 \mathrm{~min}$ at $72^{\circ} \mathrm{C}$, and then a final extension for $5 \mathrm{~min}$ at $72^{\circ} \mathrm{C}$. The methylation reactions were carried out in 1X M.SssI buffer with $160 \mu \mathrm{M}$ SAM (New England Biolabs, Ipswich, MA). In total reaction volume of $50 \mu 1,500 \mathrm{ng}$ PCR product was treated with $4 \mathrm{U}$ M.SssI for $1 \mathrm{~h}$ at $37^{\circ} \mathrm{C}$. Reactions were stopped for $20 \mathrm{~min}$ at $65^{\circ} \mathrm{C}$ and PCR product was purified Qiagen PCR purification kit (Qiagen). This $\mathrm{CpG}$ methyltransferase reaction was performed twice. Then M.SssI treated PCR product was produced (14). Curve was fitted and methylation levels were modified.

Western blot analysis. All of samples were collected and total cell lysates were prepared in M-PER mammalian protein extraction reagent (Thermo, Rockford, IL) containing a protease-inhibitor cocktail (Nalarai Tesque). Proteins $(20 \mu \mathrm{g})$ were loaded on NuPAGE $+10 \%$ Bis-Tris gels (Invitrogen Life Technologies, Carlsbad, CA) for electrophoresis. The proteins were separated at $100 \mathrm{~mA}$ for $1 \mathrm{~h}$, then transferred to polyvinylidene difluoride membranes by using iblot transfer 
for $7 \mathrm{~min}$ (Invitrogen). The membranes were incubated with Tris-buffered saline (TBS), containing 5\% non-fat milk, $0.2 \%$ Tween-20 and a rabbit anti-NR4A3 polyclonal antibody (1:100) overnight (SC-30154, Santa Cruz Biotechnology, Santa Cruz, CA). The membranes were washed three times with a TBS containing $0.2 \%$ Tween-20. The immunocomplexed proteins were identified by reaction with a peroxidase-linked goat antibody to rabbit IgG (GE Healthcare, Little Chalfont, UK). Then these immunocomplexed proteins were detected by enhanced chemiluminescent reaction (Amersham Bioscience Inc., Piscataway, NJ). Immunoblotting with antibody to actin (Abcam, Cambridge, MA) provided an internal control for equal protein loading. Chemiluminescent detection was performed by LAS4000 (Fujifilm, Tokyo, Japan).

Immunohistochemical staining. Formalin-fixed, paraffinembedded serial sections $(4 \mu \mathrm{m})$ were deparaffinized in xylene, rehydrated through graded alcohols, and immersed for $15 \mathrm{~min}$ in phosphate-buffered saline (PBS). The sections were soaked in $10 \mathrm{mmol} / \mathrm{l}$ of sodium citrate buffer ( $\mathrm{pH} \mathrm{6.9)}$ and treated in a microwave for $15 \mathrm{~min}$ for antigen retrieval. After antigen retrieval the endogenous peroxidase activity was blocked with $3 \%$ hydrogen peroxidase in methanol for $30 \mathrm{~min}$, and non-specific staining was then blocked by 1-h incubation with normal goat serum (Nichirei Biosciences). The sections were then incubated overnight at $4^{\circ} \mathrm{C}$ with $2 \mu \mathrm{g} / \mathrm{ml}$ of a rabbit anti-NR4A3 polyclonal antibody (SC-30154: Santa Cruz Biotechnology). The sections were treated for $30 \mathrm{~min}$ at room temperature with goat secondary antibody against rabbit immunoglobulins (Nichirei Biosciences). The sections were stained at room temperature for $25 \mathrm{~min}$ with AEC substrate kit (Vector Laboratories, Burlingame, CA). After staining with AEC substrate kit the sections were counterstained with hematoxylin.

Chromatin immunoprecipitation assays. Chromatin immunoprecipitation assays were performed essentially as previously described (15-17) with the following minor modifications. Neuroblastoma cell lines (NB9 and NB69) were fixed in $0.33 \mathrm{M}$ (1\%) formaldehyde for $10 \mathrm{~min}$, before adding 4 volumes of ice-cold PBS containing $0.125 \mathrm{M}$ glycine, to give an approximately 2-fold molar excess of glycine over formaldehyde. Then cells were washed with cold PBS containing Protease K (Nalarai Tesque). Crude cell lysates were sonicated to generate 200-1,000 bp DNA fragments. Chromatin was immunoprecipitated with $10 \mu \mathrm{l}$ of rabbit antiserum raised against human CTCF (07-729; Millipore, Billerica, MA) per $1 \times 10^{7}$ cells or with $2 \mu \mathrm{g}$ normal rabbit immunoglobulin $\mathrm{G}$ (IgG; Millipore) used as a control, according to manufacturer's protocols (Millipore). PCR amplification was performed using AccuPrime (Invitrogen) in a $25 \mu \mathrm{l}$ reaction volume using PCR primers at $200 \mathrm{nM}$ final concentration and $5 \mu \mathrm{l}$ immunoprecipitated DNA as a template. Amplification was carried out with an initial denaturing at $94^{\circ} \mathrm{C}$ for $1 \mathrm{~min}$ followed by 38 cycles of denaturing at $94^{\circ} \mathrm{C}$ for $30 \mathrm{sec}$, annealing for $30 \mathrm{sec}$ at the annealing temperature of each primer, extension for $1 \mathrm{~min}$ at $68^{\circ} \mathrm{C}$, and then a final extension for $5 \mathrm{~min}$ at $72^{\circ} \mathrm{C}$, using specific primers as follows: for NR4A3 exon 3, 5'-CTTCCCGCTCTTCCACTTC-3'; and 5'-TCACCTTGAAAAAGCCCTTG-3', Tm $58^{\circ} \mathrm{C}$; for cMYC, 5'-GTTTTAAGGAACCGCCTGTCCTTC-3' and 5'-GGA
TTGCAAATTACTCCTGCCTCC-3', Tm $62^{\circ} \mathrm{C}$ (18). All primers were purchased from Operon.

Statistical analysis. The Mann-Whitney U test was used to evaluate the statistical significance of the difference in the methylation level of NR4A3 among the samples. The methylation levels were categorized by Youden index using 17 patients passed the observation period (19). The cutoff point between high and low levels of DNA methylation at each DMR was calculated by ROC curve analysis. Survival curves were calculated according to Kaplan-Meier analysis and compared with a log-rank test. Event-free survival was calculated as the time from diagnosis to event or last examination if the patient had no event. Recurrence, progression of disease and death from disease were counted as events. Death resulting from therapy complications or from second malignancy was not counted as an event but censored for event-free survival. The data were analyzed by the SPSS (Chicago, IL) for Windows. Differences were considered significant at $\mathrm{p}<0.05$.

\section{Results}

Methylation levels at $\mathrm{CpG}$ sites of $\mathrm{Nr} 4 \mathrm{a} 3$ exon $3 \mathrm{CpGi}$ and its expression in mouse brain specimens. Methylation levels of each $\mathrm{CpG}$ site at the $\mathrm{Nr} 4 \mathrm{a} 3$ exon $3 \mathrm{CpG}$ island in mouse brains were analyzed at three different developmental stages. This CpGi (mouse chr4:48072571-48072905 in the USCS database, February, 2006 assembly) showed higher methylation level in the brain specimens of 12-weeks-old mice (AD brain), compared with brain specimens from 15-days-old embryos (E15) or new-born mice (NB) in the analysis using Mass ARRAY EpiTYPER (Fig. 1A). The average methylation level of all $\mathrm{CpG}$ sites in this region was significantly higher in AD brain than in E15 and NB brain specimens (Fig. 1B). Western blot analysis revealed higher expression level of NR4A3 protein in $\mathrm{AD}$ brain specimens, compared with the other developmental stages (Fig. 1C).

Search for the most different somatic change within homologous Nr4a3 exon $3 \mathrm{CpGi}$ in human neuroblastoma. We analyzed methylation level of human homologous region (chr4:48072371-48072905, in USCS genome database, March, 2006, NCBI36/hg18) in the surgical resected NB specimens and found that all $3 \mathrm{NB}$ specimens showed significantly lower methylation level at NR4A3 exon $3 \mathrm{CpGi}$ compare to those from the matched adrenal tissues (Fig. 2). The CpGi located between $N R 4 A 3$ promoter and intron 1 was not methylated at all in either neuroblastoma or adrenal samples.

Aberrant methylation at NR4A3 exon 3 CpGi confirmed by using Mass ARRAY EpiTYPER method in additional 17 neuroblastoma specimens. Additional 17 neuroblastoma samples and 1 adrenal sample were used to confirm aberrant methylation at $N R 4 A 3$ exon $3 \mathrm{CpGi}$. Adding a new adrenal sample, all the 4 adrenal samples were hypermethylated at $N R 4 A 3$ exon 3 CpGi. The average methylation level in 4 adrenal samples was $64.6 \pm 2.1 \%$. The methylation level of neuroblastoma specimens varied from $* 83.3 \pm$.. to $*-0.5 \pm 0.9 \%$, and the average value of the all samples was $27.0 \pm 25.8 \%$, which was significantly lower than the average methylation level of 4 normal samples 
A

on 1000000000 100\% Netrabled

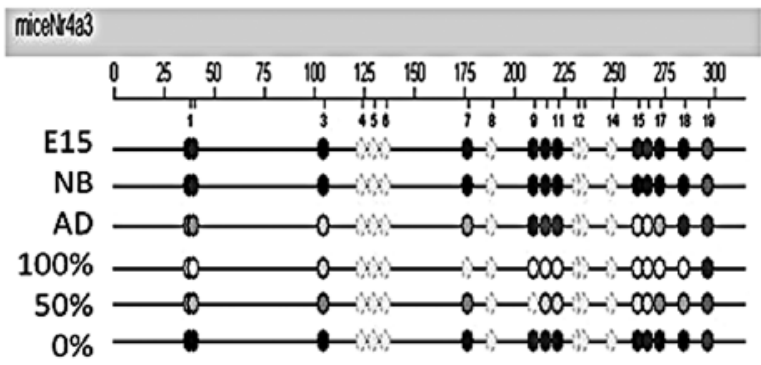

C

AD 2

NB 1

B

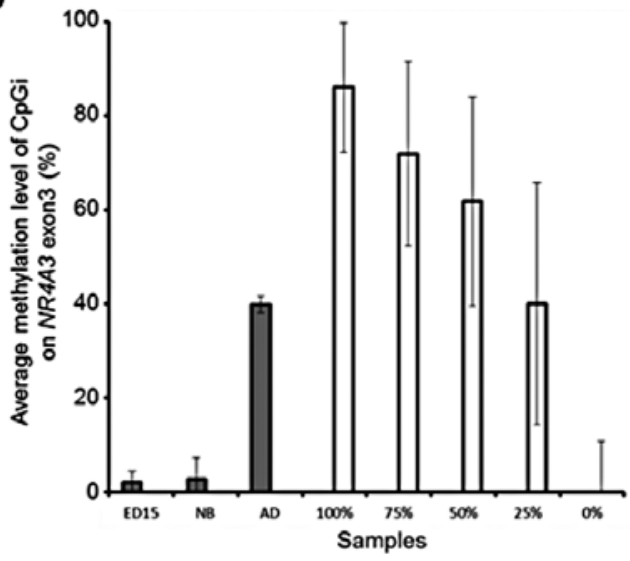

Nr4a3

\section{$\beta$-actin}

Figure 1. DNA methylation and protein expression levels of NR4A3 in mouse brain samples. DNA methylation level was analyzed quantitatively using Sequenom MassARRAY EpiTYPER. Methylation level is shown in Epigram (A) and average methylation levels are shown in the bar graph (B). Gray columns indicate mouse brain samples and open columns are the standard. Error bars indicate SD. Methylation levels in AD mouse brain samples were significantly higher than those in NB and E15 brains. (p<0.030). (C) NR4A3 and loading control of $\beta$-actin protein expression was analyzed by western blotting. AD brain showed higher expression level of NR4A3 than in NB and E15.

A

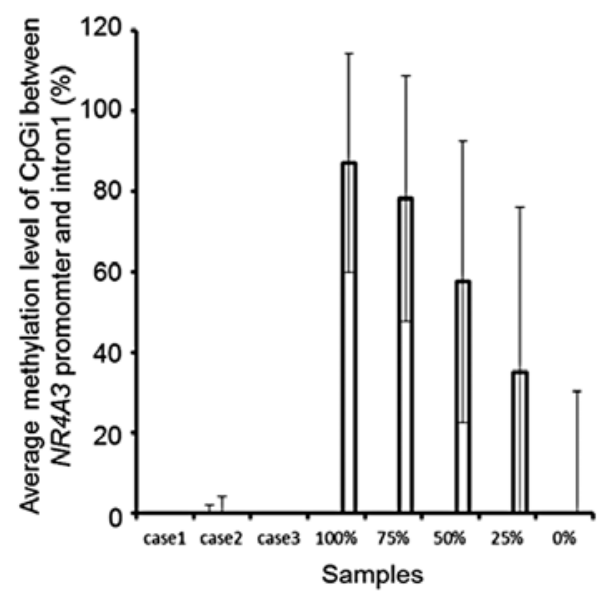

$10 \mathrm{~kb}$

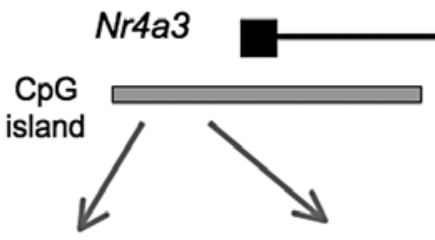

B

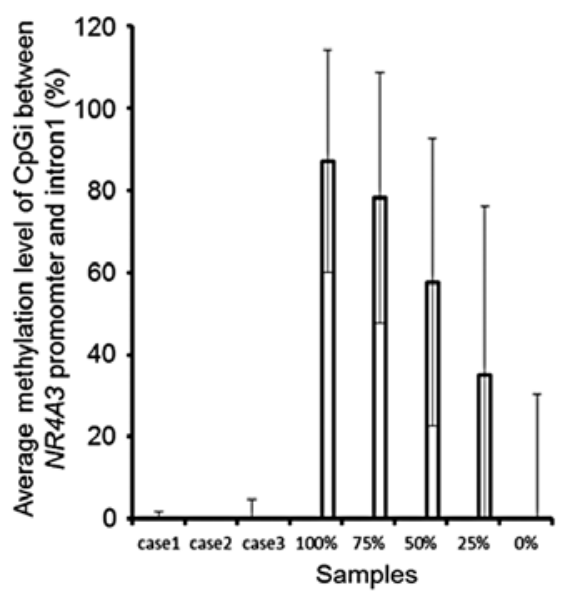

C

Figure 2. DNA methylation levels of the $N R 4 A 3$ region in human neuroblastoma specimens. Methylation levels of human homologous region of $N r 4 a 3$ were analyzed quantitatively using Sequenom MassARRAY EpiTYPER in human neuroblastoma. Methylation levels of CpGi at NR4A3 exon 3 (C), but not in 5' promoter $\mathrm{CpGi}$ (A and B), were significantly higher in neuroblastoma than those in corresponding adrenal glands (p<0.01). Gray columns indicate neuroblastoma samples, black columns are for adrenal samples and open columns are the standard. Data are shown as mean \pm SD. 
A

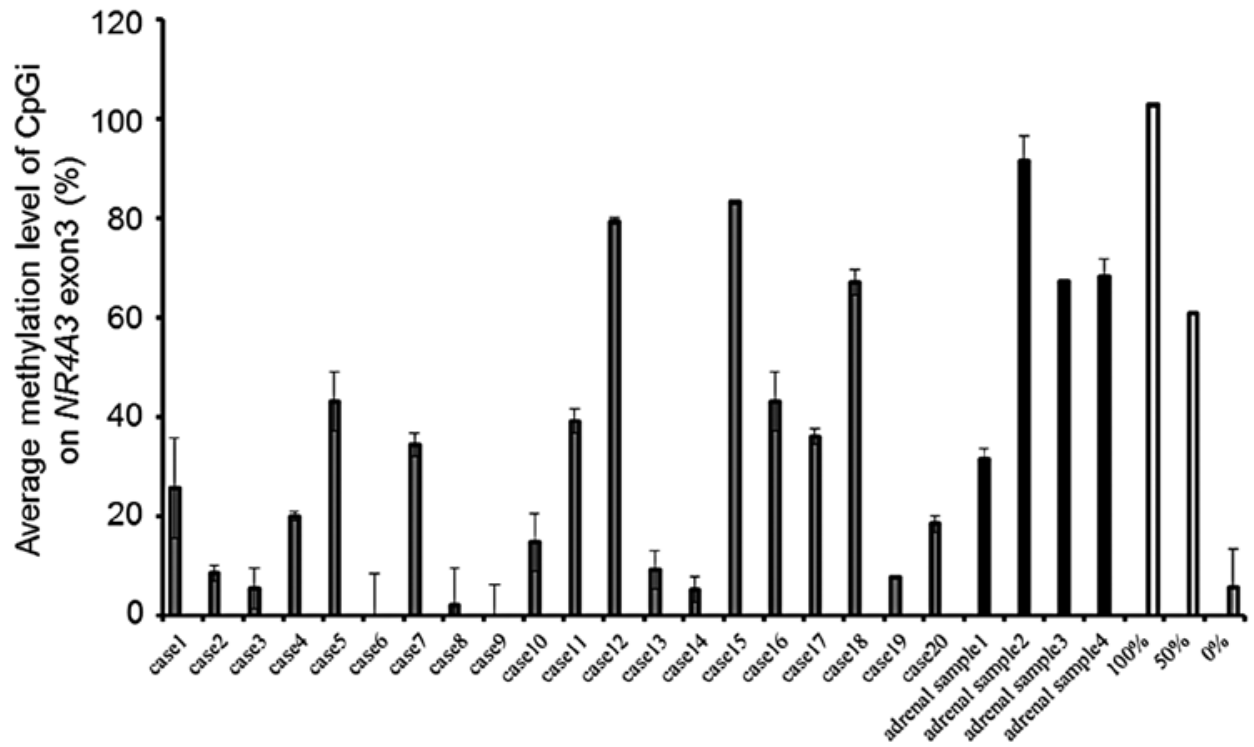

B

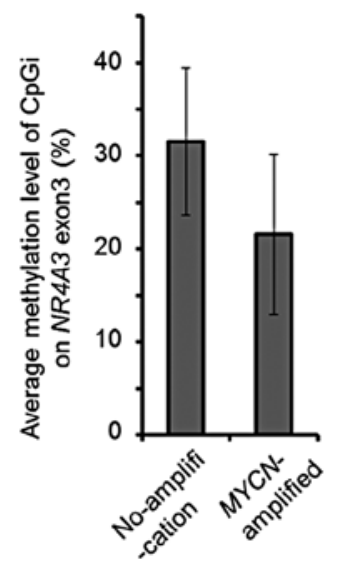

C

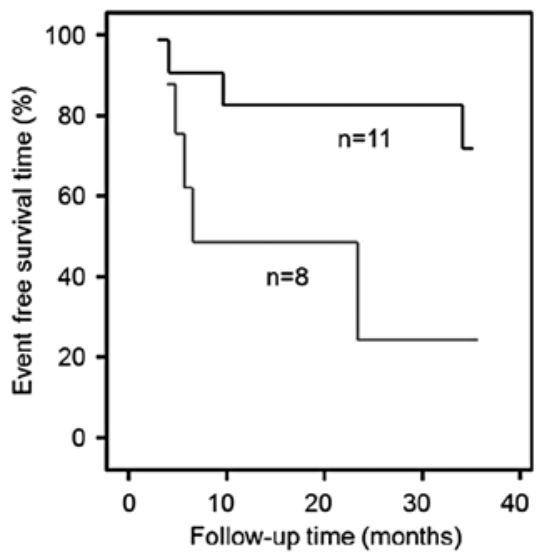

Figure 3. DNA methylation levels of $N R 4 A 3$ exon $3 \mathrm{CpGi}$ in additional human neuroblastoma specimens and survival analyses of neuroblastoma patients. Methylation levels in NR4A3 exon $3 \mathrm{CpGi}$ were analyzed by using Mass ARRAY EpiTYPER method in 20 neuroblastoma specimens and 4 adrenal samples. (A) The bar graphs show the average of methylation levels in the region. Gray columns indicate neuroblastoma samples, black columns are for adrenal samples and open columns are the standard. The error bars indicate SD. (B) Average methylation level of CpGi at NR4A3 exon 3 in 11 samples without MYCN amplification and 9 samples with MCYN amplification are shown. The error bar indicate SEM. (C) Kaplan-Meier analysis was performed to see whether methylation level at NR4A3 exon 3 CpGi associate with the survival length of neuroblastoma patients. Twenty neuroblastoma specimens were segregated into two groups depending on their methylation levels of the $N R 4 A 3$ exon 3 regions (hypermethylation, methylation level is higher than 11.93\%; hypomethylation, methylation level is $11.93 \%$ or lower). Eight out of 20 neuroblastoma specimens were in the hypomethylation tumor group. Black line indicates the hypermethylation group and gray line is for the hypomethylation group. There was a significant association between methylation levels and patient outcome ( $\mathrm{p}=0.034, \log$-rank test).

( $\mathrm{p}=0.005$, Mann-Whitney U test) (Table I, Fig. 3A). In 17 out of 20 neuroblastoma specimens, methylation levels at NR4A3 exon $3 \mathrm{CpGi}$ were low, compared with the average methylation level in 4 adrenal samples. Methylation level in 9 MYCN amplified neuroblastoma specimens was significantly lower, compared with that in 11 specimens without $M Y C N$ amplification (Fig. 3B) ( $\mathrm{p}=0.005$, Mann-Whitney U test).

For Kaplan-Meier analysis, cut off value of the methylation level was calculated as $11.93 \%$ by using youden index and the methylation levels of 20 patients passed the observation period. Twenty neuroblastoma patients were divided into two groups depending on their methylation levels at the NR4A3 exon 3 regions. The hypermethylation group has methylation level higher than the cut off value, and the hypomethylation group showed lower than that. Eight out of 20 neuroblas- toma specimens were classified to hypomethylation group. There was significant association between the methylation level at NR4A3 exon $3 \mathrm{CpGi}$ and patient outcome ( $\mathrm{p}=0.034$, log-rank test) (Fig. 3C).

Immunohistochemical staining (IHC). Immunohistochemical analysis using NR4A3 antibody showed a strong signal in adrenal tissue sections and pronounced cytoplasmic staining. On the other hand, faint staining was seen in neuroblastoma specimens (Fig. 4).

Correlation between NR4A3 exon 3 CpGi methylation and NR4A3 protein expression in neuroblastoma cells. The average methylation level at $N R 4 A 3$ exon $3 \mathrm{CpGi}$ in NB9 cells was $44 \pm 23.2 \%$, on the other hand, it was $97.1 \pm 5.8 \%$ in NB69 
A
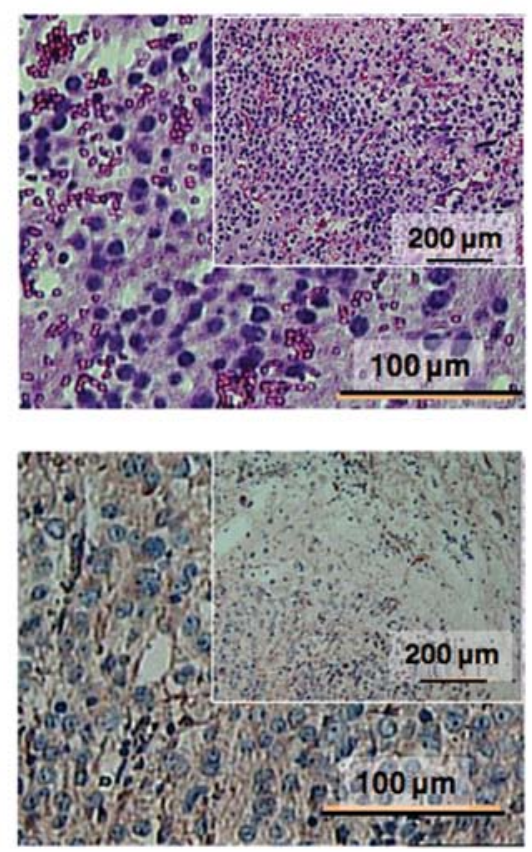

B
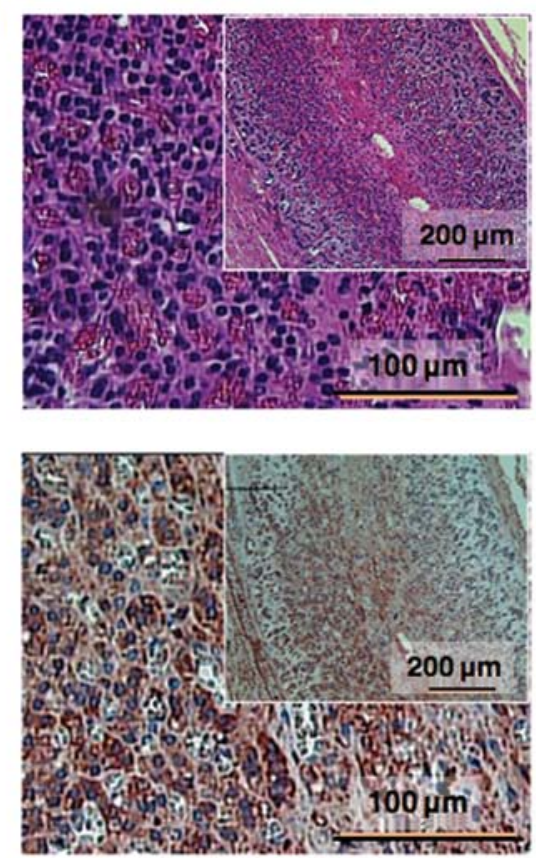

Figure 4. Imunohistochemical analyses of NR4A3 in neuroblastoma and adrenal samples. H\&E staining (upper) and immunohistochemical analysis using anti-NR4A3 antibody (lower) were performed for (A) a neuroblastoma section and (B) an adrenal section. Immune reactivity was stronger in adrenal sample than in the neuroblastoma section.

cell (Fig. 5A and B). Western blot analysis revealed higher expression level of NR4A3 protein in NB69 compared with NB9 (Fig. 5C). This result also indicates that NR4A3 protein expression was correlated with $N R 4 A 3$ exon $3 \mathrm{CpGi}$ methylation in human neuroblastoma cell lines.

Chromatin immunoprecipitation assays. We examined chromatin immunoprecipitation assays to elucidate the mechanism in which methylation level of NR4A3 exon $3 \mathrm{CpGi}$ regulates the expression level. DNA purified from the immunoprecipitated chromatin using anti-CTCF antibody was amplified by PCR for a candidate CTCF binding site of NR4A3 exon 3. In this analysis, the amplified PCR fragment was detected clearly in NB9 cells, but not in NB69 (Fig. 5D).

\section{Discussion}

Many studies have shown that epigenetic alterations, especially aberrant DNA methylation, were involved in the development of various adult tumors $(20,21)$. In neuroblastomas, aberrantly methylated genes, $64 \%$ for THBS1; $30 \%$ for TIMP-3; $27 \%$ for $M G M T ; 25 \%$ for $p 73 ; 18 \%$ for RB1; $14 \%$ for $D A P K, p 14 A R F$, p16INK4 $a$ and CASP8, respectively, and $0 \%$ for TP53 and GSTP1 have been reported and the striking differences in methylation status within neuroblastomas has suggested the existence of methylator phenotype, which might be associated with more aggressive forms of neuroblastoma $(22,23)$. Neuroblastoma development is associated with aberration of neural differentiation, and we have reported that aberrant methylation in neuroblastoma at T-/DS-DMR, which plays an important role in differentiation and development (24).

Development of neuroblastoma is related with aberration of the function of neural development factors, such as
NGF-dependent tyrosine kinase receptor TrkA activation, relating to differentiation in normal and neoplastic neuronal cells. NR4A are reported to play an important role in the development of neurons (25) and in the regulation of neural function (26). NR4A belongs to a group of early responsive genes, mediating fast response to pleiotropic extracellular stimuli. They bind to NGFI-B response element (NBRE), induce the downstream genes and affect many type of biological function such as oxidative metabolism, cell proliferation, differentiation, apoptosis and dopamine functions in the brain $(9,27)$. In view of their role in brain function, it was reported that NR4A genes, including NR4A3, were induced by psychoactive drugs such as cocaine, morphine, haloperidol and clozapine (28). In cultured cerebellar granule neurons, NR4A transcripts translocated from nucleus to mitochondria during excitotoxicity, contributing to the induction of apoptosis (29).

Our present data about mouse brains also indicate the involvement of NR4A3 and its exon $3 \mathrm{CpGi}$ methylation in the neural development. In the analysis of neuroblastoma specimens, NR4A3 exon 3 CpGi showed low methylation level in neuroblastoma compared with adrenal samples. In addition, hypermethylation of the $N R 4 A 3$ exon $3 \mathrm{CpGi}$ was significantly associated with favorable outcome. Since there was a correlation between methylation level at NR4A3 exon 3 CpGi and NORl expression, our present data suggest that NOR 1 expression level and its genome methylation could be prognostic biomarkers in neuroblastoma.

Methylation of $\mathrm{CpG}$ sites at exonic region may be linked to epigenetic remodeling of genomic DNA structure. One of the factors is CCCTC binding factor (CTCF), which is highly conserved in higher eukaryotes. CTCF binds to CTCF-binding sites, and this binding is often regulated by DNA methylation. Of the CTCF-binding sites $45 \%$ are located on intergenic, 
A

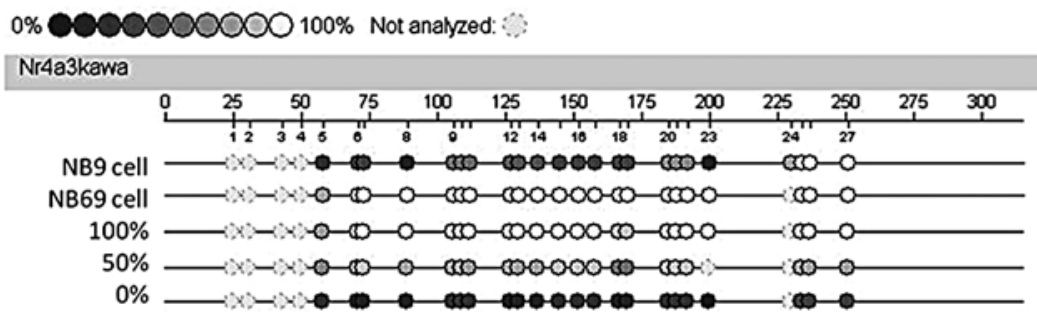

B

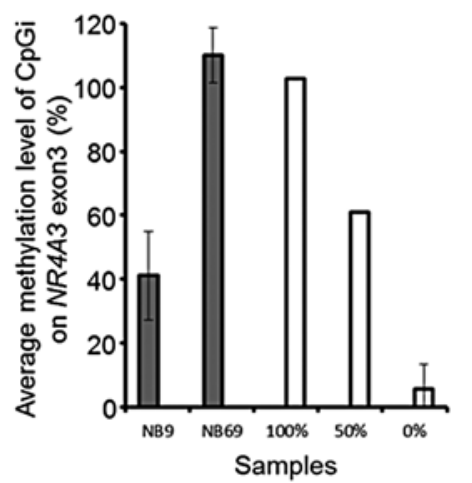

C

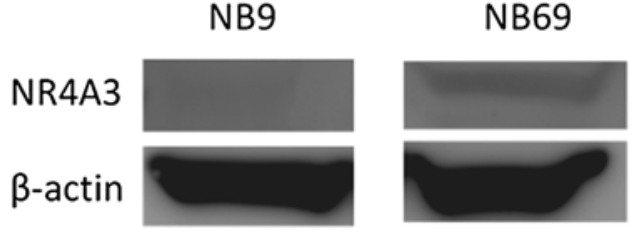

D

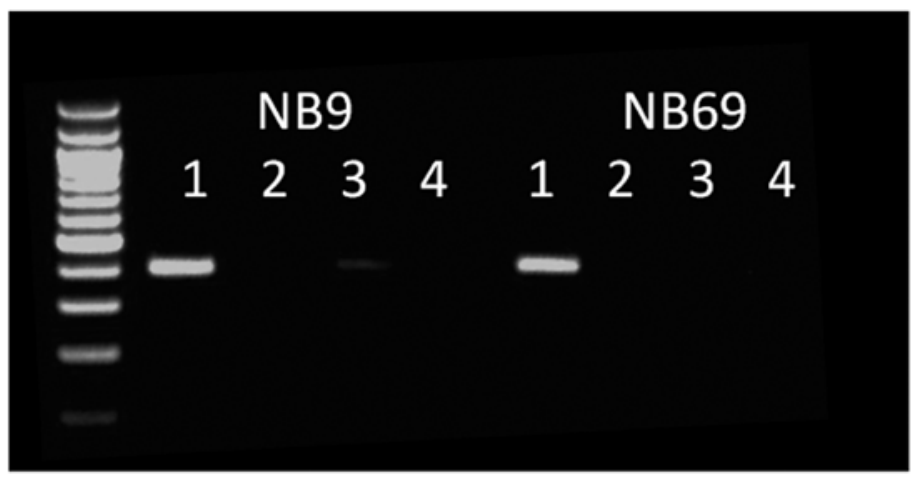

\section{1 , Input $2, \lg G$ 3, sample 4, no templete}

Figure 5. DNA methylation and protein expression levels of NR4A3 in human neuroblastoma cell lines. DNA methylation levels were analyzed quantitatively using Sequenom MassARRAY epiTYPER. (A) Methylation level of each CpG is shown in Epigram. (B) Average methylation level of each CpG site in this region is shown. Gray columns indicate neuroblastoma cell lines and open columns are the standard. Error bars indicate SD. (C) NR4A3 protein expression was analyzed by western blotting. NB69 showed high methylation level of the NR4A3 gene and expressed NR4A3 protein, while hypomethylated NB9 cells did not express the protein. (D) Chromatin immunoprecipitation assay of NR4A3 exon 3 region. Crosslinked DNA-protein complexes were immunoprecipitated with anti-CTCF antibody and control antibodies, followed by PCR amplification using primers for NR4A3 exon 3. Precipitant from NB9 lysate contained NR4A3 exon 3 region, but no product was amplified in the precipitant from NB69 lysate.

$7 \%$ 5'-untranslated region (UTR), 3\% exonic, $29 \%$ intronic and $20 \%$ within $2.5 \mathrm{~kb}$ of promoters. H19 DMR is one of CTCF-binding sites and its methylation level is related to epigenetic remodeling, which is co-localized with cohesion. Aberrant regulation of CTCF expression is associated with occurrence of cancers, such as colorectal carcinoma $(18,30,31)$. Our present study showed that CTCF can coimmunoprecipitate with a CTCF binding site within NR4A3 exon 3 CpGi in NB9 cells but not in NB69 cells. This suggests hypermethylation of NR4A3 exon $3 \mathrm{CpGi}$ in NB69 cells may inhibit its binding to the CTCF and therefore initiate expression of NOR1. This expression regulation via CTCF binding may be a mechanism of NOR1 down-regulation and involved in advanced neuroblastomas and/or neuronal de-differentiation.
This is the first report indicating that DNA methylation level of NR4A3 exon $3 \mathrm{CpGi}$ is associated with NOR1 expression and aberrantly methylated in neuroblastomas. In addition, our present data suggest that hypermethylation of this region might be one of the prognostic factors in this tumor. Although further analyses are necessary to elucidate the regulation mechanism of NOR1 expression and its function, our present data strongly suggest that this gene could be involved in neural differentiation and human neuroblastomagenesis.

\section{Acknowledgements}

This study was supported by Nihon University Multidisciplinary Research Grant for 2006 (to H.N.), the Academic Frontier 
Project for 2006 Project for Private Universities, a matching fund subsidy from MEXT (to H.N.), National Cancer Institute Grant CA102423 (to W.A.H and H.N.), National Cancer Institute Center Support Grant CA16056 (to Roswell Park Cancer Institute) and Grant-in-Aid from the Ministry of Education, Science, Sports and Culture of Japan (grant nos. 20592092 and 23300344).

\section{References}

1. De Bernardi B, Nicolas B, Boni L, Indolfi P, Carli M, Cordero Di Montezemolo L, Donfrancesco A, Pession A, Provenzi M, di Cataldo A, Rizzo A, Tonini GP, Dallorso S, Conte M, Gambini C, Garaventa A, Bonetti F, Zanazzo A, D'Angelo P and Bruzzi P: Disseminated neuroblastoma in children older than one year at diagnosis: comparable results with three consecutive high-dose protocols adapted by the Italian Co-Operative Group for Neurobalstoma. J Clin Oncol 21 : 1592-1601, 2003

2. Matthay KK, Villablanca JG, Seeger RC, Stram DO, Harris RE, Ramsay NK, Swift P, Shimada H, Black CT, Brodeur GM, Gerbing RB and Reynolds CP: Treatment of high-risk neuroblsatoma with intensive chemotherapy, radiotherapy, autologous bone marrow transplantation, and 13-cis-retinoic acid. Children's Cancer Group. N Engl J Med 341: 1165-1173, 1999.

3. Wang H, Lee S, Nigro CL, Lattanzio L, Merlano M, Monteverde M, Matin R, Purdie K, Mladkova N, Bergamaschi D, Harwood C, Syed N, Szlosarek P, Briasoulis E, McHugh A, Thompson A, Evans A, Leigh I, Fleming C, Inman GJ, Hatzimichael E, Proby C and Crook T: NT5E (CD73) is epigenetically regulated in malignant melanoma and associated with metastatic site specificity. Br J Cancer 106: 1446-1452, 2012.

4. Geiman TM and Muegge K: DNA methylation in early development. Mol Reprod Dev 77: 105-113, 2010.

5. Bird A: DNA methylation patterns and epigenetic memory. Genes Dev 16: 6-21, 2002.

6. Weber M, Hellmann I, Stadler MB, Ramos L, Pääbo S, Rebhan M and Schübeler D: Distribution, silencing potential and evolutionary impact of promoter DNA methylation in the human genome. Nat Genet 39: 457-466, 2007.

7. Song F, Smith JF, Kimura MT, Morrow AD, Matsuyama T, Nagase $\mathrm{H}$ and Held WA: Association of tissue-specific differentially methylated regions (TDMs) with differential gene expression. Proc Natl Acad Sci USA 102: 3336-3341, 2005.

8. Eckhardt F, Lewin J, Cortese R, Rakyan VK, Attwood J, Burger M, Burton J, Cox TV, Davies R, Down TA, Haefliger C, Horton R, Howe K, Jackson DK, Kunde J, Koenig C, Liddle J, Niblett D, Otto T, Pettett R, Seemann S, Thompson C, West T, Rogers J, Olek A, Berlin K and Beck S: DNA methylation profiling of human chromosomes 6, 20 and 22. Nat Genet 38: 1378-1385, 2006.

9. Zhao Y and Bruemmer D: NR4A orphan nuclear receptors: transcriptional regulators of gene expression in metabolism and vascular biology. Arterioscler Thromb Vasc Biol 30: 1535-1541, 2010.

10. Maxwell MA and Muscat GE: The NR4A subgroup: immediate early response genes with pleiotropic physiological roles. Nucl Recept Signal 4: e002, 2006.

11. Song F, Mahmood S, Ghosh S, Liang P, Smiraglia DJ, Nagase H and Held WA: Tissue specific differentially methylated regions (TDMR): Changes in DNA methylation during development. Genomics 93: 130-139, 2009.

12. Li LC and Dahiya R: MethPrimer: designing primers for methylation PCRs. Bioinformatics 18: 1427-1431, 2002.

13. Ehrich M, Nelson MR, Stanssens P, Zabeau M, Liloglou T, Xinarianos G, Cantor CR, Field JK and van den Boom D: Quantitative high-throughput analysis of DNA methylation patterns by base-specific cleavage and mass spectrometry. Proc Natl Acad Sci USA 102: 15785-15790, 2005.

14. Fatemi M, Pao MM, Jeong S, Gal-Yam EN, Egger G, Weisenberger DJ and Jones PA: Footprinting of mammalian promoters: use of a CpG DNA mehtyltransferase revealing nucreosome positions at a single molecule level. Nucleic Acids Res 33: e176, 2005.
15. Baxter EW, Cummings WJ and Fournier REK: Formation of a large, complex domain of histone hyperacetylation at human 14q32.1 requires the serpin locus control region. Nucleic Acids Res 33: 3313-3322, 2005.

16. Orlando V, Strutt H and Paro R: Analysis of chromatin structure by in vivo formaldehyde cross-linking. Methods 11: 205-214, 1997.

17. Bowers SR, Mirabella F, Calero-Nieto FJ, Valeaux S, Hadjur S, Baxter EW, Merkenschlager M and Cockerill PN: A conserved insulator that recruits CTCF and cohesin exists between the closely related but divergently regulated interleukin-3 and granulocyte-macrophage colony-stimulating factor genes. Mol Cell Biol 29: 1682-1693, 2009.

18. Ishihara K, Oshimura $\mathrm{M}$ and Nakao M: CTCF-dependent chromatin insulator is linked to epigenetic remodeling. Mol Cell 23: 733-742, 2006.

19. Akoben AK: Understanding diagnostic tests 3: Receiver operating characteristic curves. Acta Paediatr 96: 644-647, 2007.

20. Egger G, Liang G, Aparicio A and Jones PA: Epigenetics in human disease and prospects for epigenetic therapy. Nature 27: 457-463, 2004.

21. Laird PW: Cancer epigenetics. Hum Mol Genet 15: 65-76, 2005.

22. Gonzalez-Gomez P, Bello MJ, Lomas J, Arjona D, Alonso ME, Amiñoso C, Lopez-Marin I, Anselmo NP, Sarasa JL, Gutierrez M, Casartelli C and Rey JA: Aberrant methylation of multiple genes in neuroblastic tumours. relationship with MYCN amplification and allelic status at 1p. Eur J Cancer 39: 1478-1485, 2003.

23. Banelli B, Vinci AD, Gelvi I, Casciano I, Allemanni G, Bonassi S and Romani M: DNA methylation in neuroblastic tumors. Cancer Lett 228: 37-41, 2005.

24. Kawashima H, Sugito K, Yoshizawa S, Uekusa S, Furuya T, Ikeda T, Koshinaga T, Shinojima Y, Hasegawa R, Mishra R, Igarashi J, Kimura M, Wang X, Fujiwara K, Gosh $S$ and Nagase H: DNA hypomethylation at the ZNF206-exon 5 CpG island associated with neuronal differentiation in mice and development of neuroblastoma in humans. Int $\mathbf{J}$ Oncol 40: 31-39, 2012.

25. Zetterström RH, Williams R, Perlmann T and Olson L: Cellular expression of the immediate early transcription factors Nurrl and NGFI-B suggests a gene regulatory role in several brain regions including the nigrostriatal dopamine system. Brain Res Mol Brain Res 5: 111-120, 1996.

26. Ohkura N, Ito M, Tsukada T, Sasaki K, Yamaguchi K and Miki K: Structure, mapping and expression of a human NOR-1 gene, the third member of the Nur77/NGFI-B family. Biochim Biophys Acta 1308: 205-214, 1996.

27. Eells JB, Wilcots J, Sisk S and Guo-Ross SX: NR4A gene expression is dynamically regulated in the ventral tegmental area dopamine neurons and is related to expression of dopamine neurotransmission genes. J Mol Neurosci 46: 545-553, 2012.

28. Werme M, Ringholm A, Olson L and Brené S: Differential patterns of induction of NGFI-B, Norl and c-fos mRNAs in striatal subregions by haloperidol and clozapine. Brain Res 863: 112-119, 2000.

29. Boldingh Debernard KA, Mathisen GH and Paulsen RE: Differences in NGFI-B, Nurr1, and NOR-1 expression and nucleocytoplasmic translocation in glutamate-treated neurons. Neurochem Int 61: 79-88, 2012.

30. Wendt KS, Yoshida K, Itoh T, Bando M, Koch B, Schirghuber E, Tsutsumi S, Nagae G, Ishihara K, Mishiro T, Yahata K, Imamoto F, Aburatani H, Nakao M, Imamoto N, Maeshima K, Shirahige K and Peters JM: Cohesin mediates transcriptional insulation by CCCTC-binding factor. Nature 451: 796-801, 2008.

31. Phillips JE and Corces VG: CTCF: master weaver of the genome. Cell 137: 1194-1211, 2009. 